\title{
Informe de pasantía de extensión: Desarrollo de las prácticas docentes en LEBEM en el periodo 2005(I) - 2012 (I) (sistematización de unidades didácticas de practica intermedia V) ${ }^{1}$
}

\author{
Report of pasantía of extensión: Desarrollo de las practices \\ teachers en en el LEBEM period 2005 (I) - 2012 (I) (systematization \\ of didactic units of practica intermedia $\mathrm{V}$ ) \\ Relatório de Estágio de Extensão: Desenvolvimento de práticas \\ de ensino LEBEM no período de 2005 (I) - 2012 (I) (unidades \\ de ensino prática sistemática de intermediário V)
}

Recibido: enero de 2012

Aceptado: mayo de 2012
Andrea Paola Torres Chaparro ${ }^{2}$

Sonia Yisel Mora González ${ }^{3}$ Christian Andrés Rodríguez Ramírez ${ }^{4}$

\section{Resumen}

En la licenciatura en Educación Básica con Énfasis en Matemáticas se concibe la práctica como eje articulador del conocimiento profesional del profesor, dichas prácticas han sufrido cambios conforme a las necesidades que surgen con el desarrollo de la formación ciudadana y los objetos matemáticos, invitando a los EPM a la reflexión en torno a los cinco énfasis definidos para cada espacio de formación; para mirar el desarrollo de la práctica intermedia $\mathrm{V}$ con énfasis en diseño curricular, se parte de la recolección y sistematización de las unidades didácticas realizadas por los EPM durante los periodos dispuestos, logrando hacer el análisis preliminar de la información puesta en las guías del profesor y protocolos y su relación con los demás elementos constitutivos.

Palabras clave: Profesor; formación de profesores; práctica; fases; recolección; sistematización.

\section{Abstract}

In the Bachelor of Primary Education with Emphasis in Mathematics is seen as the linchpin practice of teachers' professional knowledge, such practices have changed according to the needs that arise with the development of civic education and mathematical objects, inviting the EPM to reflection on the five focus defined for each training area, to look at the development of intermediate $\mathrm{V}$ practice with emphasis on curriculum development, is part of the collection and systematization of the teaching

1 Articulo de Investigación.

2 Universidad Distrital Francisco José de Caldas. Bogot;a, Colombia. Contacto: andypaol@hotmail.com

3 Universidad Distrital Francisco José de Caldas. Bogot;a, Colombia. Contacto: yiyiton16@hotmail.com

4 Universidad Distrital Francisco José de Caldas. Bogot;a, Colombia. Contacto: cardenal093@hotmail.com. 
units made by EPM during periods willing, managing to make a preliminary analysis of the information placed in the teacher's guides and protocols and their relationship with other components.

Keywords: Teacher, teacher training, practice, phases, collection, systematization.

\section{Resumo}

No Bacharelado em Educação Básica com Ênfase em Matemática é visto como a prática pivô do conhecimento profissional dos professores, tais práticas têm mudado de acordo com as necessidades que surgem com o desenvolvimento da educação cívica e objetos matemáticos, convidando o EPM a reflexão sobre a cinco foco definido para cada área de formação, de olhar para o desenvolvimento da prática intermediária $\mathrm{V}$ com ênfase no desenvolvimento do currículo, faz parte da recolha e sistematização das unidades de ensino feitas por EPM durante os períodos dispostos, conseguindo fazer uma análise preliminar das informações colocadas em guias e protocolos e sua relação com outros componentes do professor.

Palavras-chave: Professor, formação de professores, prática, fases, coleta, sistematização.

\section{Presentación del problema}

En la licenciatura en educación básica con énfasis en matemáticas se ha definido la práctica docente como el eje integrador del conocimiento profesional del profesor, es por ello que en el aula el estudiante para profesor de matemáticas puede reformar los planteamientos bajo los que fue educado, promoviendo la construcción de los objetos matemáticos y contribuyendo a la formación ciudadana, Lurduy (2010) resalta la importancia de adoptar un modelo de profesor crítico, reflexivo, investigador, constructivo, plural diverso y complejo en el aula.

La sistematización de las experiencias de formación, dadas a partir del análisis y la organización de las guías para profesor y protocolos de las unidades didácticas elaboradas por los estudiantes para profesor, reflejan como se pone en juego el conocimiento base para la enseñanza de los objetos matemáticos respecto a la relación entre conocimiento práctico y razonamiento pedagógico, el cual es necesario para el diseño y análisis de la implementación correspondiente, además dicha sistematización busca valorar el accionar, las interpretaciones y el desarrollo de las practica, por ello se espera contribuir en esta etapa de la investigación a la organización recolectando las unidades realizadas por los estudiantes para profesor de matemáticas (EPM) en la práctica intermedia $\mathrm{V}$ en el periodo 2005 (I) a 2012 (I) y realizando un análisis preliminar que permita la reducción de las unidades encontradas conforme a diferentes categorías.

\section{Marco de referencia}

El Perfil del estudiante para profesor en el proyecto LEBEM se encamina a reconocer las herramientas, posiciones y criterios con los que cuenta y debería contar un EPM a la hora de diseñar las guías para profesor. De acuerdo a lo estipulado por el Grupo de Práctica Docente del proyecto curricular LEBEM (2011):

Los instrumentos metodológicos utilizados en la práctica tendrán que ver con el diseño de la secuencia didáctica de actividades de aula en las cuales se 
incluyen: los contenidos básicos a trabajar, los procesos o procedimientos y los contextos, los referentes teóricos básicos, las estrategias metodológicas, los recursos didácticos a utilizar, las estrategias de evaluación (tanto grupal como individual) y los organizadores del currículo escolar. Con la aplicación de la secuencia didáctica de las actividades de aula se lleva a cabo también la evaluación de la gestión en el aula, todo ello con el fin de desarrollar el razonamiento didáctico de los futuros profesores a la vez que iniciarlos en los procesos de investigación en el aula. (p.5).

Los EPM tienen su primer acercamiento a la propuesta de planeación del grupo DECA (1992), dicha propuesta cimienta sus bases en un modelo constructivista, la propuesta DECA es un modelo de enseñanza que permite establecer el desarrollo e implementación de una secuencia didáctica, establecida mediante fases que permite constituir objetivos, características y roles implicados en el proceso de enseñanza aprendizaje, en ella se encuentran las actividades de enseñanza-aprendizaje en cuatro bloques; Iniciación e introducción, desarrollo y reestructuración, aplicación y profundización y evaluación, por su parte con el transcurrir de las prácticas se pasa a propuestas de planeación a través de la teoría de las situaciones didácticas de Brousseau la cual se apoya en la modelización del proceso instructivo, usando para su sustento conceptos que describen el proceso de construcción del conocimiento del estudiante en compañía del profesor.

En relación a las consideraciones anteriores, el proyecto curricular LEBEM manifiesta que dentro de la planeación del trabajo de aula es importante que la propuesta contenga de acuerdo a lo señalado por Guerrero y otros (2006) mínimo los siguientes aspectos: formulación del problema, antecedentes, hipótesis, pregunta orientadora, objetivos generales y específicos, marco de referencia teórico, metodología de clase, referencias.

Para realizar la sistematización y análisis preliminar se determina una base a través de la teoría fundamentada, dicha teoría según De la Torres y otros (s.f) es un método de investigación no lineal, el cual realiza un análisis de cada uno de los métodos constitutivos de los textos, los cuales son capturados y analizados por medio de la investigación cualitativa, los elementos constitutivos de la teoría fundamentada deben ser escritos y detallados, por su importancia para la comprensión de los procesos de investigación, dichos elementos son los siguientes: el muestreo teórico, incidentes, saturación teórica, método comparativo constante (m.c.c.), categorías, categoría central, las familias, codificación y categorización, tipos de codificación, codificación abierta, memos, la teoría sustantiva y la teoría formal- En cuanto a la metodología se considera que esta teoría es una teoriza generadora, lo que significa que al ser de tipo espiral, los datos van surgiendo y va surgiendo con la integración de teoría a través de métodos comparativos, sobre los datos obtenidos durante el trascurso de la investigación, lo que hace que se cree un esquema teórico emergente, que surge a través de una clasificación teórica, que a su vez se da por medio de la recolección sistemática de datos sobre el campo de trabajo.

\section{Metodología}

Se espera contribuir en esta etapa de la investigación a la organización y análisis preliminar de la información, recolectando las unidades realizadas por los EPM en la práctica intermedia $\mathrm{V}$ en el periodo 2005 (I) a 2012 (I), En función de la recolección, organización y análisis de las unidades didácticas de la practica intermedia $\mathrm{V}$ con énfasis en diseño y bajo los planteamientos anteriores, surge la siguiente pregunta orientadora:

¿De qué manera la recolección, organización, sistematización y análisis preliminar de las unidades didácticas contribuyen a la caracterización del desarrollo del saber pedagógico de contenido en la práctica $\mathrm{V}$ con énfasis en diseño curricular durante los periodos 2005(I) a 2012 (I)?

Objetivo. Recolectar, organizar y realizar análisis preliminar de la información puesta en las guías de profesor y protocolos de algunas Unidades Didácticas (UD) elaboradas por los EPM de las 
Tabla 1

\begin{tabular}{|c|c|c|}
\hline Fase & Tarea & Actividad \\
\hline Exploratoria & $\begin{array}{l}\text { Asistencia a las sesiones de } \\
\text { capacitación llevadas a cabo } \\
\text { por los investigadores del } \\
\text { proyecto. } \\
\text { Elaboración de fichas } \\
\text { bibliográficas y descriptivas } \\
\text { sobre las seis lecturas } \\
\text { asignadas para comprender } \\
\text { el proyecto de investigación } \\
\text { aprobado nor el IEIE }\end{array}$ & $\begin{array}{l}\text { Lectura de los documentos para } \\
\text { realizar fichas bibliográficas y } \\
\text { descriptivas según el modelo de } \\
\text { análisis documental de Consuelo de } \\
\text { Hoyos. }\end{array}$ \\
\hline $\begin{array}{l}\text { Apropiación y profundización } \\
\text { teórica metodológica }\end{array}$ & $\begin{array}{l}\text { Selección de unidades didacticas de } \\
\text { práctica V. } \\
\text { Construir el marco teórico específico } \\
\text { y metodológico que posibilite la } \\
\text { construcción de criterios para la } \\
\text { recolección y organización de la } \\
\text { información. }\end{array}$ & $\begin{array}{l}\text { Revisión y recolección en el banco } \\
\text { de unidades didácticas disponibles en } \\
\text { Documentación de LEBEM y dialogo } \\
\text { con los profesores que tuvieron a } \\
\text { cargo los espacios de formación en los } \\
\text { periodos respectivos }\end{array}$ \\
\hline $\begin{array}{l}\text { Construcción de los instrumentos } \\
\text { para la recolección, organización y } \\
\text { análisis preliminar de los datos }\end{array}$ & $\begin{array}{l}\text { Construir parrillas para vaciar } \\
\text { información como tablas, esquemas, } \\
\text { diagramas. } \\
\text { Usar y seleccionar algunas técnicas } \\
\text { del análisis de contenido planteando } \\
\text { discusiones en torno a lo que dice las } \\
\text { teorías de codificación como la Teoría } \\
\text { Fundamentada en los datos, para la } \\
\text { reducción de la muestra de algunas } \\
\text { unidades didácticas de practica cinco } \\
\text { en el periodo } 2005 \text { (I) - } 2012 \text { (I) }\end{array}$ & $\begin{array}{l}\text {-Lecturas horizontales y } \\
\text { verticales de unidades } \\
\text { didácticas } \\
\text {-Pilotaje y validación de } \\
\text { datos recogidos en cada } \\
\text { unidad por categorías para } \\
\text { sugerir posible organización } \\
\text { de los datos. }\end{array}$ \\
\hline $\begin{array}{l}\text { Análisis preliminar de la } \\
\text { información contenido en las } \\
\text { unidades didácticas }\end{array}$ & $\begin{array}{l}\text { Proponer sistema de codificación } \\
\text { en cada categoría para el análisis } \\
\text { horizontal y vertical de las unidades } \\
\text { didácticas. }\end{array}$ & $\begin{array}{l}\text { Construcción de ideas principales por } \\
\text { categoría. }\end{array}$ \\
\hline
\end{tabular}

Fuente: Elaboración propia

práctica intermedia $\mathrm{V}$, entre los periodos del 2005 (I) a 2012 (I), de manera que se contribuya a identificar el desarrollo del saber pedagógico de contenido puesto en juego por los EPM vinculado al énfasis de diseño curricular.
Plan de Acción y formación

En este plan de acción y formación se hace evidente cada una de las etapas a desarrollar en la pasantía, resaltando las tareas y actividades, es importante 
en la metodología del trabajo destacar la apropiación teórica, seguida de la recopilación de unidades didácticas y su análisis a través de diferentes filtros descritos en cada fase.

\section{Conclusiones}

Una vez puesto en marcha el plan de acción y formación se pasa a la fase explotaría en la cual se realiza el conocimiento y apropiación de la investigación y se definen los elementos teóricos puestos en juego durante la pasantía, en la fase de apropiación se realiza la recolección de 93 unidades didácticas de práctica $V$ en los periodos 2005(I)-2012(I), sin encontrar unidades del periodo 2008 (I) y 2008 (III), para poder decantar las unidades se definieron los criterios de coherencia, pertinencia, validez, decantando las unidades que serán revisadas para identificar el desarrollo del saber pedagógico de contenido.

\section{Referencias}

De la Torres, G. Di Carlo, E. Santana, A. Carvajal, H. Ramírez, C. Rodríguez, P. Sánchez, A. Tirado, J. (s.f). Teoría fundamentada. Madrid. Universidad autónoma de Madrid

Grupo DECA. (1992). Orientaciones para el diseño y elaboración de actividades de aprendizaje y evaluación. España.

Guerrero, F. Sánchez, N \& Lurduy, O (2006). La práctica docente a partir de los Modelos DECA y TSD. Bogotá. Universidad Distrital.

Guerrero, F. Sánchez, N \& Lurduy, O (2011). La práctica docente en el proyecto curricular LEBEM. Bogotá. Universidad Distrital. 